\title{
Mobile learning como estrategia innovadora en el aprendizaje de la química inorgánica
}

\section{Mobile learning as an innovative strategy in the learning of inorganic chemistry}

SOSA, Jorge A. ${ }^{1}$

RODRIGUEZ, Ariel A. ${ }^{2}$

ALVAREZ, William $0^{3}$

FORERO, Aracely. ${ }^{4}$

\section{Resumen}

El aprendizaje en el área de química en ocasiones es complejo, algunos estudiantes no alcanzan el desempeño escolar básico, razón por la cual se innovó su enseñanza empleando aplicaciones didácticas Apps para dispositivos móviles, con el fin de motivar al estudiante en la adquisición del conocimiento. El proceso investigativo de este estudio se enmarca en el método cuantitativo con enfoque descriptivo y diseño correlacional, se concluyó que la integración de recursos digitales como las Apps permiten mejorar el aprendizaje de los educandos y que estos alcancen altos niveles en su desempeño escolar.

Palabras clave: mobile learning, estrategia didáctica, química inorgánica

\begin{abstract}
Learning in the area of chemistry is sometimes complex, some students do not reach basic school performance, which is why their teaching was innovated using didactic applications Apps for mobile devices, in order to motivate the student to acquire knowledge; the investigative process of this study is framed in the quantitative method with descriptive focus and correlational design, it was concluded that the integration of digital resources such as Apps allow to improve the learning of the students and that they reach high levels in their school performance.

key words: mobile learning, teaching strategy, inorganic chemistry
\end{abstract}

\section{Introducción}

Tradicionalmente los estudiantes han sentido apatia hacia el aprendizaje de algunas disciplinas científicas como la física y la química. En los escenarios educativos se escuchan frases como: que clase tan aburrida, no me gusta esta asignatura, es demasiado monótona, entre otras; se ha evidenciado que la raíz de esta problemática no es

\footnotetext{
${ }^{1}$ Grupo de investigación TICA. Universidad Pedagógica y Tecnológica de Colombia. Profesional en Quimica de alimentos, Magister en TIC Aplicadas a las Ciencias de la Educación. Universidad Pedagógica y Tecnológica de Colombia. E- mail: jorge.sosa@uptc.edu.co

${ }^{2}$ Grupo de investigación TICA y TELEMATICS Research Group. Universidad Pedagógica y Tecnológica de Colombia. Profesor Maestría en TIC Aplicadas a las Ciencias de la Educación. Doctor (c) Tecnología Educativa. Magister Software Libre. Ingeniero de Sistemas. Email: ariel.rodriguez@uptc.edu.co ${ }^{3}$ Grupo de investigación SIMILES. Universidad Pedagógica y Tecnológica de Colombia. Licenciado en informática para la docencia, Esp. Ingenieraia del Software, Magister en TIC Aplicadas a las Ciencias de la Educación. Universidad Pedagógica y Tecnológica de Colombia. E- mail: William_orla_nd@hotmail.com

${ }^{4}$ Grupo de investigación SIMILES. Universidad Pedagógica y Tecnológica de Colombia. Doctora en Multimedia Educativa, Directora y profesora Maestría en TIC aplicadas a las Ciencias de la Educación, Universidad Pedagógica y Tecnológica de Colombia. E-mail: Aracely.forero@uptc.edu.co
} 
en sí el conocimiento de estas áreas, sino la manera en que didácticamente son articuladas. Los educadores se han arraigado al método de enseñanza tradicional centradose en la catedra magistral y han dejado de lado los estilos de aprendizaje de los educandos. Es decir, consideran que todos aprenden de la misma manera, pero la realidad es otra, pues cada estudiante es un mundo diferente con características particulares en su forma de aprender. Por tanto, si no se innovan los procesos educativos, particularmente la didáctica, se puede incurrir en la monotonia que puede converger en el bajo rendimiento escolar de los educandos.

En relación al aprendizaje de la química como área del currículo escolar Furió (2006) argumenta que los docentes identifican falta general de interés de los educandos por esta disciplina del conocimiento, percepción que ha sido corroborada por los propios estudiantes en diferentes trabajos de investigación. En efecto, señalan como principales causantes de su actitud desfavorable, de su desinterés hacia el aprendizaje, la enseñanza de una ciencia descontextualizada de la sociedad y de su entorno, poco útil y sin temas de actualidad, junto a otros factores como los métodos de enseñanza de los profesores, los cuales califican de aburridos y poco participativos, la escasez de prácticas $y$, especialmente, la falta de recursos didácticos que sean acordes a sus intereses y expectativas. (p.222)

Igualmente, Cantú (1999) reseña que existen diversas razones para explicar las dificultades que presentan los educandos en la comprensión y desarrollo de contendidos o conceptos en la asignatura de química, señalando entre ellas:

- El aprendizaje de la química inorgánica "suele ser aburrido o confuso, convirtiéndose así en una limitación para el aprendizaje de esta ciencia".

- Habitualmente los estudiantes caen en el memorismo para solo pasar un examen, y olvidar todo al momento después de haber salido de él.

- Son contados los estudiantes que logran adquirir el lenguaje químico con facilidad, pero a la gran mayoría de ellos se les dificulta.

En este sentido, Díaz (2010) afirma que es necesario innovar las estrategias didácticas, interpretando la innovación como el resultado de la incorporación de las novedades educativas del momento. Dentro de este marco se incluyen las nuevas tecnologías que están en auge y más importante aún, que son atractivas para los adolescentes, por cuanto despiertan su curiosidad e interés para el desarrollo de competencias básicas del área, propiciando espacios reflexivos en el aula y ofreciendo a los estudiantes mayores elementos que contribuyan a fortalecer su aprendizaje y gusto por áreas como la química.

Particularmente a través de aplicación de un diagnóstico desarrollado con estudiantes de grado décimo del colegio Guilermo León Valencia ubicado en el municipio de Duitama, Boyacá, Colombia, se corroboran los postulados de los autores, pues se identificó que lo estudiantes no sienten gusto en el aprendizaje de la química inorgánica. Por esta razón se realizó un estudio en el que se integraron aplicaciones móviles (Apps) para ser empleadas como estrategia de innovación en la enseñanza del tema grupos funcionales inorgánicos a través de terminales móviles como tablets y celulares. Se buscó resignificar el modelo de enseñanza tradicional permitiendo que el estudiante dejara de ser receptor de contenidos y se convirtiera en agente que interviene en su proceso de aprendizaje, es decir, que tuviera el protagonismo en la construcción del conocimientoIgualmente se buscó a través de la integración de estos recursos tecnológicos facilitar el desarrollo de la labor del docente, permitiendole incorporar nuevas didácticas que fortalezcan el proceso enseñanza - aprendizaje e igualmente transformen la figura del educador al convertirse en guía y orientador del aprendizaje mas que trasnmisor de información. 


\section{1. ¿Qué es la química?}

Nakamatsu (2012) indica que la química es una ciencia que intenta explicar las propiedades macroscópicas de la materia a partir de su estructura conformada por entidades submicroscópicas (partículas). Así, a partir de objetos concretos y visibles la química crea conceptos y abstracciones, y forma modelos que presentan una interpretación de la naturaleza para dar una visión coherente de la realidad. Para lograr esta interpretación, se definen las partículas básicas: los átomos, que a su vez pueden formar entidades más complejas como compuestos iónicos y moléculas, a través de la formación de enlaces químicos. Pero estos modelos no solo permiten a la química describir el mundo en que vivimos, sino que pueden además predecir la formación y propiedades de materia no existente. Así, esta disciplina no solo es capaz de comprender la materia que existe en la naturaleza sino que también crea nueva materia. (p.38)

\subsection{El porqué de la química en el contexto educativo}

Sin lugar a dudas nuestro entorno se encuentra integrado por diferentes sustancias y materiales que contribuyen a mejorar la calidad de vida de las personas, razón por la cual la química cobra relevancia, dado que la mayor parte de elementos $u$ objetos que se emplean en la cotidianidad están sometidos a procesos químicos a través de los cuales se transforman para llegar a un estado funcional, por ejemplo, los alimentos que se ingieren contienen preservantes que retardan su deterioro, se utilizan fertilizantes y plaguicidas para mejorar la eficiencia de los cultivos, el sistema de transporte está basado en combustibles como la gasolina y el diésel (o biodiésel). La comodidad en los hogares la brindan materiales poliméricos como los plásticos, pinturas, barnices, espumas elásticas. Los artefactos que se utilizan diariamente contienen piezas hechas de plásticos, metales o materiales cerámicos, que, a su vez, han requerido de procesos químicos para su fabricación. Los avances en la medicina están basados en productos y procesos químicos.

Deboer (2000) sostiene que el estilo de vida moderno genera nuevos problemas como el calentamiento global, el agujero en la capa de ozono, la contaminación del aire en las grandes ciudades, la gran cantidad de desechos que se generan, la calidad del agua, entre otros, la química como ciencia puede ser parte de la solución a estos problemas. Por tanto, es importante que los estudiantes posean un conocimiento mínimo de esta disciplina de las ciencias, por un lado, para tener un entendimiento básico de cómo funcionan las cosas a su alrededor, y de esta forma comprender los descubrimientos y problemas que desafían a la ciencia y la sociedad en la actualidad. $Y$, por otro lado, que puedan ser sujetos intervinientes en la toma de decisiones fundamentadas y responsables sobre los problemas en el mundo. Esto es lo que algunos investigadores consideran que se debe realizar desde los escenarios educativos y a lo que han denominado alfabetización científica. Además, al margen del contenido científico de un curso de química, los conocimientos que adquieren los educandos desde esta disciplina del saber contribuye con el fortalecimiento y desarrollo de sus habilidades intelectuales, mejorando su capacidad de conceptualizar, de manejar ideas nuevas, de utilizar simbolismos y enriquecer sustancialmente su vocabulario. (p.590)

\subsection{Porqué el rechazo a la Química por parte de los estudiantes}

Hernández y Montagut (1991) en su investigación ¿Qué sucedió con la magia de la Química?, identificaron que uno de los factores que incide en la disminución del interés de los estudiante por esta área del conocimiento, si no el principal, es la forma en que los docentes abordan la enseñanza de esta ciencia. Los cursos de química generalmente están sobrecargados con material teórico, y muy orientados hacia los principios y teorías. Además, se le da mucha importancia a la resolución de problemas numéricos artificiales, y muy poca a las reacciones químicas, que son el corazón de esta ciencia. Por otro lado, se aborda en primer lugar el estudio de los aspectos microscópicos de la materia, y se posponen los aspectos fenomenológicos. 
Las autoras reseñan que dicho método de enseñanza conllevó a que la química, fuese perdiendo su carácter atractivo y motivador para los estudiantes, razón por la cual los educandos no aprenden nada acerca de la fascinación de hacer algo nuevo y creativo con la química, pues se les presenta como una colección de principios más o menos abstractos, que aparentemente no tienen ninguna relevancia práctica en su mundo cotidiano.

En la actualidad existen dos tendencias a nivel mundial, una de ellas plantea volver a enseñar la química fenomenológica y vivencial y otra enseñar los principios en los que se basa (estructura, fisicoquímica, cinética, entre otros). El ideal sería que se aborde la enseñanza de la química a través de los fenómenos, para que en cursos superiores, los educandos puenden entender modelos que expliquen la realidad antes observada. Por otro lado es importante hacer notar que en la selección de contenidos y metodologías, se debe tomar en cuenta la madurez y capacidad de abstracción de la mayoría de los estudiantes. Si los estudiantes no han alcanzado la etapa del pensamiento formal, es más adecuado para lograr un mejor aprendizaje, enfocar la enseñanza desde una perspectiva fenomenológica. Primero la experiencia en el laboratorio y más tarde la abstracción. (Hernández y Montagut,1991,p.2)

Al respecto Izquierdo (2004) argumenta que en las aulas, la enseñanza de la química se debe orientar desde fenómenos relevantes y significativos, las experiencias escolares deben garantizar una dinámica que permita a los educandos pensar, hacer y comunicarse de manera coherente de acuerdo a las leyes de esta disciplina. Es crucial presentar a los estudiantes las teorías apropiadas a sus conocimientos y a las prácticas experimentales que puedan llegar a realizar, esto no es fácil y supone un profundo replanteamiento para identificar los obstáculos a superar de tal manera que se de cumplimiento a los propósitos y metas de aprendizaje. (p.120)

Fernández y Moreno (2008) desde sus estudios, establecen que la dificultad del aprendizaje en el área de química por parte de los educandos, se asocia a una imagen abstracta al estar fundamentada en átomos a los que no se tiene acceso, y al lenguaje simbólico que se emplea y que es ajeno al que conocen y utilizan estos en su cotidianidad. Incluso el objeto de la química (describir y comprender las propiedades de las sustancias y los intercambios de materia) queda alejado de los intereses de los educandos que suelen aceptar los fenómenos más llamativos sin intentar comprenderlos. (p.5)

\subsection{Utilización de las TIC en la enseñanza y aprendizaje de la Química}

A pesar de que los métodos empleados para la enseñanza de la química se han centrado en el uso de recursos didácticos de orden convencional, en las dos últimas décadas con la integración de las tecnologías de la información y la comunicación, se ha buscado resignificar la enseñanza de diferentes áreas y asignaturas del currículo escolar. Particularmente se ha querido dar un giro a la enseñanza de la química integrando recursos y medios Ilamativos como la multimedia que emplea: imágenes, videos, sonidos, entre otros. Cabero (2007) indica que el uso de las TIC en esta disciplina del conocimiento posibilita en los estudiantes la capacidad de examinar, interactivamente y en tres dimensiones, las moléculas de un compuesto; realizar prácticas de laboratorio virtuales; y conseguir en Internet toda clase de información para sus investigaciones. Es por esto, que el hecho de encontrar imágenes de compuestos o reacciones químicas minimiza el problema en su aprendizaje, que radica en explicar un mundo microscópico en uno macroscópico, siendo el uso de estas herramientas tecnológicas un medio eficaz para entender los fenómenos químicos y lograr un mejor proceso de aprendizaje por parte de los educandos.

En particular Cabero (2007) señala que para fortalecer el proceso enseñanza - aprendizaje en el área de química, se pueden emplear tres grupos de recursos digitales, el primero de ellos hace relación al uso de Internet con el cual se puede encontrar información para la presentación y desarrollo de actividades del área, en el segundo grupo se encuentra los laboratorios y simuladores virtuales, los cuales se pueden emplear considerando que uno de los objetivos significativos que persigue la enseñanza de la química, es la realización de prácticas de 
laboratorios; y el uso de estas herramientas ofrece una serie de posibilidades y ventajas que en muchas ocasiones llegan a ser la dificultad de una práctica real, finalmente en el tercer grupo se encuentran los recursos de aprendizaje colaborativo, entre ellos los webquest, los blogs y las wiki, recursos que cada vez son más utilizados y requieren la participación del usuario para la construcción conjunta del conocimiento.

No obstante, en los últimos años con la evolución tecnológica, los dispositivos móviles han incorporado sistemas que permiten el empleo de recursos didácticos para la educación, un ejemplo de ello son los sistemas operativos Android e IOS, que facilitan el uso de apliaciones móviles Apps para innovar el aprendizaje con metodologias como el Mobile Learning o aprendizaje móvil, en el cual el educando sin importar el lugar o ubicación geográfica puede tener acceso a la información, aplicando los principios de la ubicuidad. Este tipo de aprendizaje ha cobrado relevancia, teniendo en cuenta que los jóvenes se ineteresan en el empleo del celular en su cotidianidad. Pofr esta razón, el estudio presentado da a conocer una experiencia educativa en la que se hizo uso de esta clase de tecnología para potenciar el aprendizaje de los grupos funcionales inorgánicos, tema del área de química en estudiantes de grado décimo de educación secundaria.

\section{Mobile Learning}

El m-Learning, es un espacio relativamente nuevo de aprendizaje, que se encuentra en continuo progreso y crecimiento, fruto de la evolución tecnológica y pedagógica, se cree que esta metodología desciende del elearning, entendiendo a este último como todo tipo de difusión del conocimiento educativo a través de Internet, lo que hace que el $\mathrm{m}$-Learning sea catalogado como una variante del e-Learning.

Quinn (2000) define el m-Learning como el simple aprendizaje que se lleva a cabo con la ayuda de dispositivos móviles, o la intersección de la informática móvil (la aplicación de pequeños, portátiles y dispositivos de informática y de las comunicaciones inalámbricas). De acuerdo con esta definición, autores como Turunen, Syvaenen, Ahonen (2003) ven los dispositivos móviles como una estrategia didáctica que puede fortalecer el aprendizaje de los educandos al permitirles combinar su tiempo libre con el estudio y llegar a un nivel significativo en la aprehensión del conocimiento. Además en este nuevo modelo de aprendizaje se da la ausencia de limitaciones espacio temporales, la portabilidad de los dispositivos, el acceso ubicuo y la conectividad social, con lo cual en definitiva, se tiene la posibilidad que el aprendizaje ocurra en cualquier lugar y momento, incluyendo los entornos de aprendizaje tradicionales, tales como las aulas, pero también en otras localizaciones, como el hogar, espacios sociales e incluso durante los desplazamientos.

\subsubsection{Características y ventajas del Mobile Learning en la educación}

Castaño y Cabero (2013) reseñan que el Mobile Learning aplicado en el aprendizaje ofrece diferentes ventajas a los sujetos de la educación, por un lado el docente adquiere el rol de guia y orientador de sus educandos, y estos a su vez dejan de ser simples receptores de información y asumen un papel protagónico en la construcción del conocimiento. Las ventajas de este modelo de aprendizaje son diversas por lo cual los autores refieren entre otras:

- La flexibilidad del aprendizaje oferta la posibilidad de aprender en cualquier espacio y tiempo, siempre y cuando se disponga de un dispositivo móvil

- Proporciona una gran cantidad de recursos educativos

- Su sencillez y las facilidades que oferta hacen del Mobile Learning una técnica atrayente que muchos utilizan de forma intuitiva

- La gran conectividad de la que disponen hoy en día los dispositivos móviles ayuda a que este tipo de aprendizaje sea una realidad factible 
- El aprendizaje personalizado logra que el educando sea capaz de tomar sus propias decisiones

- La portabilidad es un factor clave en esta metodología que hace referencia a la movilidad tanto de los Smartphone como de los educandos y que incrementa notablemente el aprendizaje de éstos.

Por lo anterior, se puede considerar que el Mobile Learning se trata de un aprendizaje que maneja el propio educando, y que en muchas ocasiones lo hace dependiente de sus necesidades. Es un aprendizaje que genera múltiples ventajas (Kearney, Schuck, Burden, y Aubusson, 2012) que benefician a todas las partes e incrementan el valor de la docencia, dado que:

- Facilita la colaboración, interacción y comunicación entre estudiantes, logra que estos desarrollen habilidades y destrezas que les serán de gran uso para su futuro académico y posteriormente su desempeño laboral.

- Al tratarse de un modo personalizado de aprender, los educadores pueden crear materiales adaptados a las diversas necesidades del grupo de clase.

- El educando aumenta su motivación e interés por las asignaturas de clase al sentirse capaz de relacionar su vida cotidiana con su aprendizaje. Esto le ayuda a comprender de un mejor modo la sociedad en la que vivimos, y a construir hábitos necesarios de aprendizaje y estudio que no existían hasta el momento.

- El trabajo con Smartphones y tablets presenta verdaderas ventajas ya que son los propios estudiantes quienes se encargan de su renovación y su mantenimiento, situación que no se da cuando se trabaja con ordenadores personales.

Brazuelo y Gallego (2011) en relación al Mobile Learning indican que los estudiantes logran incrementar su atención, interés y motivación, además de sentirse conectados con su estilo de vida. Se sienten más valorados y esto incrementa su autonomía e iniciativa, llegando en la mayoría de casos a aumentar su rendimiento académico y personal, igualmente, este aprendizaje mejora el acceso e integración de los estudiantes con discapacidad o necesidades especiales. Por otro lado, los profesores dejan de verse estancados en el modo de impartir los contenidos en el aula, aumentando sus posibilidades de continuar aprendiendo. Se convierten en personas más confiadas y creativas, con una mayor capacidad de comunicación y colaboración entre compañeros. Al mismo tiempo, las instituciones educativas no se quedan atrás, a parte de adquirir un amplio conocimiento y manejo de las TIC y obtener una mejora de los recursos disponibles, logran aumentar su prestigio.

Con base en las características y ventajas que ofrece el Mobile Learning a la educación, se tiene que vale la pena desarrollar experiencias en las cuales se integre este tipo de aprendizaje, para fortalecer el desempeño académico de los educandos; particularmente seria benefico implementar el uso de aplicaciones Apps para innovar el aprendizaje del área de química y desarraigarse de los métodos de educación tradicional, que son los que no han permitido que los educandos sientan agrado y motivación para aprender las tematicas que desde esta disciplina del conocimiento son abordadas en las aulas escolares.

\subsubsection{Integración del Mobile Learning en el aula escolar}

La innovación del proceso educativo, resulta cautivante para los educandos. Por ello si se trata de vincular el Mobile Learning en las actividades escolares, es necesario contar los requisitos necesarios para ponerlo en práctica. No solo basta con disponer de dispositivos móviles necesarios y de la última tecnología en la institución educativa, sino que es necesario instruir a los estudiantes en el uso de estos dispositivos dentro del aula de clase. (Ng y Nicholas, 2013,p.710) 
Desde los postulados de Arce (2013) la integración del Mobile Learning en el aprendizaje implica defender el uso del Smartphone en el aula, utilizando los recursos didácticos adecuados para ello, considerando que las tablets suponen también una buena opción, actualmente algunos centros educativos no disponen de recursos económicos para invertir en ellas, mientras que la mayoría de estudiantes sí disponen de un teléfono móvil personal, al respecto Depetris, Travela y Castro (2012) sugieren que el empleo del celular en las actividades escolares sea regulado con base en normas y acuerdos establecidos entre docentes y estudiantes, con lo cual se pueden alcanzar las metas y objetivos de aprendizaje propuestos.

\subsubsection{Beneficios del Mobile Learning para los estudiantes}

En el mundo moderno con las tecnologias de la información emergentes, el aprender se convierte en una actividad más interesante y divertida, los recursos educativos móviles y las posibilidades que ofrecen en el trabajo colaborativo, contribuyen a simplificar actividades que antes eran complicadas de desarrollar o demandaban una dedicación excesiva. Los educandos encuentran un aliado en el aprendizaje móvil, ya que pueden aprende sin tensiones, a su ritmo, de forma más relajada, con la capacidad de repetir y reforzar aspectos en los que tienen dificultades. Hay que mencionar, además que el aprendizaje móvil como indica Chamocho (2016) ofrece a los educandos una variada gama de beneficios entre los cuales destacan: Se mejora la experiencia de aprendizaje, el aprendizaje se hace más independiente, los educandos alcanzan mejor desempeño y más motivación, se mejora la evaluación y retroalimentación del estudiante, se potencia la comunicación, las relaciones y el trabajo colaborativo, promueve y facilita actividades de investigación y se tiene mejor acceso a la información y nuevas posibilidades de encontrarla.

En consecuencia el aprendizaje móvil puede contribuir a mejorar el rendimiento escolar de los educandos a través del acceso a una variada cantidad de recursos y aplicaciones educativas innovadoras, igualmente flexibiliza la labor de docentes y estudiantes atendiendo a las necesidades particulares de cada centro educativo para dar cumplimiento a sus objetivos particulares.

\section{Metodología}

El estudio realizado se orientó desde el método de investigación cuantitativa, pues se buscó estimar la variable rendimiento académico en el área de química inorgánica de grado décimo. En relación al enfoque se siguieron los lineamientos de la investigación descriptiva, a través de la cual se interpretaron diversas situaciones que tienen que ver con el aprendizaje de los educandos, las características que estos presentan respecto a las actividades que se realizan y las estrategias didácticas que son utilizadas para la enseñanza del área referida. El diseño del estudio se orientó desde la investigación correlacional para establecer la relación que existe entre el Mobile learning como estrategia de aprendizaje y el rendimiento académico del área de química inorgánica en estudiantes de grado décimo, para este propósito se realizaron pruebas pre test y post test de conocimientos del área, para luego analizarlas, contrastarlas y establecer comparaciones a través del uso de pruebas de la estadística inferencial.

\subsection{Objetivo general}

Integrar el Mobile Learning como estrategia de enseñanza, en el área de química inorgánica de grado decimo y establecer la relación que existe entre el uso de este método y el rendimiento escolar de los estudiantes

\subsection{Hipótesis}

Con base al diseño del estudio, partiendo de la relación que se puede dar entre el rendimiento escolar de los estudiantes en el área de química y el empleo del Mobile Learning como estrategia de enseñanza se planteó la hipótesis nula de estudio: 
Hi: El uso del Mobile Learning como estrategia de enseñanza en el área de química inorgánica de grado décimo, permite que los educandos sientan agrado por los contenidos desarrollados y tengan un mejor rendimiento académico.

Teniendo en cuenta que es un estudio correlacional y que se trata de probar si la correlación que se da entre las variables es positiva o negativa se plantea la hipótesis alternativa:

Ho: El uso del Mobile Learning como estrategia de enseñanza en el área de química inorgánica de grado décimo, no resulta motivante para los educandos, por lo cual su rendimiento académico no presenta diferencias significativas.

\subsection{Variables de estudio}

Las variables empleadas en este estudio son las siguientes:

Cuadro 1

Operacionalización

Sistema de Variables

\begin{tabular}{|c|c|c|c|}
\hline VARIABLES & INDICADORES & PREGUNTAS & INSTRUMENTO \\
\hline \multirow{2}{*}{$\begin{array}{l}\text { DEPENDIENTE: } \\
\text { Rendimiento } \\
\text { escolar en el área } \\
\text { de química }\end{array}$} & $\begin{array}{l}\text { Conocimientos de los } \\
\text { estudiantes en el tema } \\
\text { grupos funcionales } \\
\text { inorgánicos }\end{array}$ & \multirow[t]{2}{*}{$\begin{array}{c}\text { ¿Cuál es el conocimiento que poseen los } \\
\text { estudiantes de grado décimo respecto al } \\
\text { tema de química inorgánica grupos } \\
\text { funcionales? }\end{array}$} & \multirow{3}{*}{$\begin{array}{c}\text { Pre Test de } \\
\text { diagnóstico } \\
\text { Encuesta de opinión } \\
\text { Observación } \\
\text { Participativa } \\
\text { Post Test de } \\
\text { conocimientos } \\
\text { Encuesta de opinión } \\
\text { Sobre el uso del } \\
\text { Mobile Learning } \\
\text { como estrategia de } \\
\text { aprendizaje }\end{array}$} \\
\hline & $\begin{array}{c}\text { Conceptualización, } \\
\text { clasificación, } \\
\text { nomenclatura y aplicación }\end{array}$ & & \\
\hline $\begin{array}{l}\text { INDEPENDIENTE: } \\
\text { Empleo del } \\
\text { Mobile learning } \\
\text { como estrategia } \\
\text { de enseñanza y } \\
\text { aprendizaje en } \\
\text { el área de } \\
\text { química } \\
\text { inorgánica }\end{array}$ & $\begin{array}{l}\text { Mejoras significativas en } \\
\text { el proceso enseñanza - } \\
\text { aprendizaje }\end{array}$ & $\begin{array}{l}\text { ¿Cómo perciben los estudiantes de } \\
\text { grado décimo el empleo del Mobile } \\
\text { Learning como estrategia para } \\
\text { fortalecer el aprendizaje del área de } \\
\text { química inorgánica? }\end{array}$ & \\
\hline
\end{tabular}

Fuente: elaboración propia

\subsection{Población}

La población objeto de estudio estuvo integrada por los estudiantes del grado décimo del colegio Guillermo León Valencia, ubicado en el municipio de Duitama, Boyacá, Colombia. Como muestra se seleccionaron treinta estudiantes correspondientes al grado 10-05, como se detalla en el cuadro 2. 
Cuadro 2

Población objeto de estudio

\begin{tabular}{ll}
\hline Criterio & Descripción \\
\hline Nivel escolar & Grado 10 de educación Media \\
\hline Género & Mujeres : $12 \quad$ Hombres: 18 \\
\hline Edad promedio & Años: 15 a 18 años \\
\hline Contexto escolar & Urbano \\
\hline Estrato Económico & 1 y 2 \\
\hline Nivel de acceso a las TIC & Bueno: La mayoría de los educandos dispone de dispositivos \\
& móviles, asimismo la institución educativa cuenta con la \\
infraestructura requerida.
\end{tabular}

Fuente: elaboración propia

\subsection{Etapas del estudio}

El estudio desarrollado se estructuro en tres etapas que permitieron dar cumplimiento a los objetivos propuestos:

Primera etapa: En esta etapa del estudio como punto de partida se aplicó a los estudiantes un test de diagnóstico, con el propósito de establecer sus conocimientos previos inherentes al tema grupos funcionales inorgánicos: Oxidos, hidróxidos, ácidos y sales, cada uno de estos temas fue evaluado desde aspectos como: definición y características, formulación, nomenclatura, usos y aplicaciones. La prueba realizada se calificó en una escala valorativa de 1.0 a 5.0, donde el primer indicador corresponde a un nivel bajo y 5.0 a un nivel superior de desempeño académico. Los puntajes alcanzados por los estudiantes se relacionan en el apartado de resultados

Segunda etapa : Con base en los resultados de la etapa diagnóstica se pudo establecer que el rendimiento escolar de los estudiantes se ubica en un nivel bajo, se identificó que estos mayormente presentan dificultades en el reconocimiento de los componentes de una moléculase evidenció , asimismo, que existen problemas en la identificación del nombre de diferentes compuestos lo cual en química inorgánica se denomina formulación y nomenclatura de grupos funcionales. Tomando como referente el rendimiento escolar de la prueba diagnostica se decidió orientar la enseñanza de la química desde la integración de las tecnologías de la información y la comunicación, particularmente el empleo del Mobile Learning como estrategia didáctica y por esta razón se construyeron unidades didacticas de los temas referidos. La experiencia de uso de las TIC abarcó un espacio de 10 sesiones de clase cada una de ellas de 4 horas semanales, es decir un total de 40 horas de clase, en las cuales se alternó el modelo de aprendizaje tradicional con el empleo del Mobile Learning, para poder establecer cual de los dos métodos de enseñanza es más adecuado para los educandos.

Tercera etapa : La tercera etapa del estudio se centró en establecer si el empleo del Mobile Learning como estrategia de aprendizaje en el área de química inorgánica, permite que los estudiantes tengan mejor desempeño académico. Para esto se aplicó una prueba de conocimientos post test inherente a las unidades didácticas mediadas por las TIC y las actividades realizadas desde el método de aprendizaje tradicional, se contrastaron las calificaciones obtenidas en ambos métodos de enseñanza basándose en la prueba pre test en relación a la prueba post test. 


\section{Resultados y discusión}

Los resultados del estudio se orientan al desarrollo de cada una de las etapas referidas en el diseño metodológico

\subsection{Etapa de diagnóstico}

En esta etapa se aplicó un test de conocimientos acerca de los grupos funcionales inorgánicos, el cual estuvo integrado por veinte ítems, y abarcó los temas: Oxidos, hidróxidos, ácidos y sales; para la valoración de la prueba se tomó como referente el sistema institucional de evaluación mostrado en el cuadro 3.

Cuadro 3

Sistema institucional de evaluación

Colegio Guillermo León Valencia

\begin{tabular}{lc} 
Calificación cualitativa & Calificación cuantitativa \\
\hline Bajo & 1.0 a 3.0 \\
\hline Básico & 3.1 a 3.9 \\
\hline Alto & 4.0 a 4.5 \\
\hline Superior & 4.6 a 5.0 \\
\hline
\end{tabular}

Fuente: elaboración propia.

Se pudo establecer que el puntaje mínimo alcanzado por los educandos en el test aplicado fue 1.0 y el máximo 2.4 como se muestra en el cuadro 4.

\section{Cuadro 4}

Resumen descriptivo de las puntuaciones del diagnóstico pre-test

\begin{tabular}{cccccc}
\hline Min. & $\mathbf{1}$ cuartil. & Mediana & Media & 3 cuartil & Máximo \\
\hline 1.00 & 0.65 & 1.5 & 1.43 & 2.1 & 2.4 \\
\hline
\end{tabular}

Fuente: elaboración propia

De acuerdo a estos resultados, se evidencia que los estudiantes tienen problemas en la identificación de los grupos funcionales inorgánicos. Al revisar el cuestionario aplicado se encontraron falencias en la escritura de la estructura de los compuestos químicos, identificación del nombre de algunos ácidos, hidróxidos y oxidos, el uso que se da en la vida real a estos compuestos y su origen o procedencia. El promedio de calificaciones alcanzó una puntuación de 1.43 en una escala de 1.0 a 5.0, razón por la cual se afirma que el desempeño académico de los educandos cualitativamente se ubica en un nivel bajo.

Respecto a este desempeño escolar, los educandos refieren que no sienten gusto en el aprendizaje del área, aducen que el método empleado por el docente y los recursos didácticos con los que se desarrollan las actividades no son adecuados; argumentan que el docente titular utiliza la catedra magistral y no permite la construcción conjunta del aprendizaje. Además de centrarse en el empleo de recursos convencionales de aprendizaje como: tablero, marcador y textos guias, igualmente, no se desarrollan actividades como talleres o experiencias de laboratorio en las cuales a través de la interacción y colaboración se llegue a adquirir el conocimiento y por ende ir más allá de un aprendizaje temporal o mermoristico.

En esencia se pudo identificar que el origen del bajo rendimiento escolar de los educandos radica en el método de enseñanza empleado por los educadores y la didáctica poco innovadora y motivante., Ôr este motivo es necesario resignificar el proceso pedagógico de la enseñanza de esta área, propendiendo motivar al educando para que pueda dar cumplimiento a los logros y metas de aprendizaje establecidos en el proyecto educativo institucional (P.E.I). 


\subsection{Etapa de diseño e implementación del Mobile Learning como estrategia didáctica}

En esta etapa con base en el diagnóstico se identificó la necesidad de implementar ambientes innovadores de aprendizaje, para resignificar la didáctica en la enseñanza del área de química inorgánica. Al tratarse de un estudio realizado desde el programa de Maestría en TIC aplicadas a las Ciencias de la Educación, teniendo como referente que el propósito de este programa académico es innovar los ambientes de aprendizaje, se precisó integrar el Mobile Learning como estrategia didáctica para mejorar la situación diagnosticada. Además la selección de dicha estrategia obedece a la disponibilidad de recursos tecnológicos que tiene la institución educativa y los estudiantes, estos últimos en su gran mayoría poseen dispositivos moviles y se les facilita el uso de esta clase de tecnologías en cualquier espacio geográfico.

Particularmente la estrategia seleccionada se estructuró a través de unidades didácticas en las cuales se integraron aplicaciones (Apps) para dispositivos móviles, entre ellas: Tabla periodica 2020 - Quimica, formulación química Lite, Los ácidos, iones y sales inorgánicos, Nomenclatura de química- Compuestos inorgánicos, Nomenclatura química inorgánica y fórmulas, juego Quiz - compuestos químicos y química master - química básica.

Figura 1

Actividades interactivas a través de las aplicaciones móviles seleccionadas

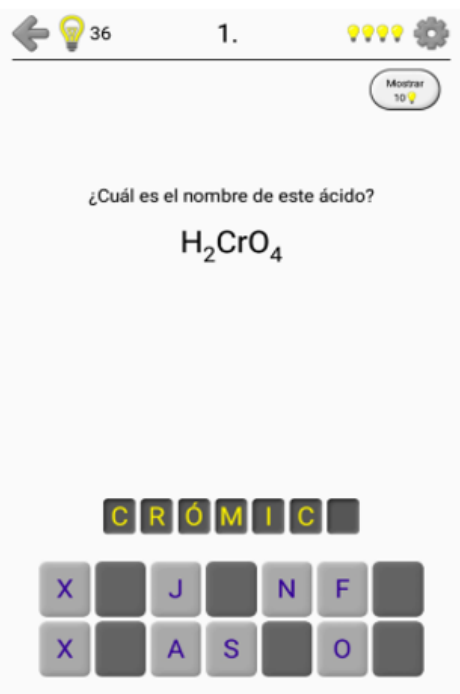

Formula :

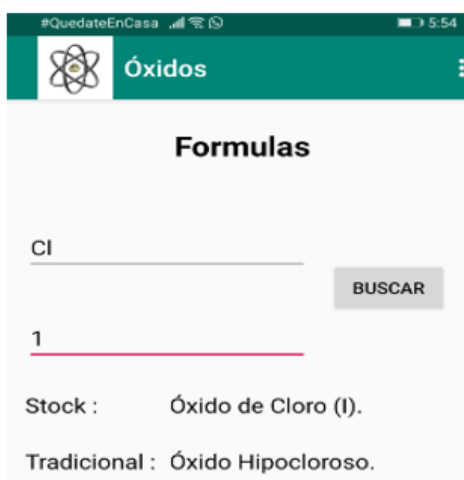

Sistematica : Monóxido de Dicloro.

$$
\begin{aligned}
& \mathrm{Cl}^{-2}+\mathrm{O}^{2} \\
& \mathrm{Cl} \times{ }^{-2} \mathrm{O} \\
& \mathrm{Cl}_{2} \mathrm{O}_{1} \\
& \mathrm{Cl}_{2} \mathrm{O}
\end{aligned}
$$

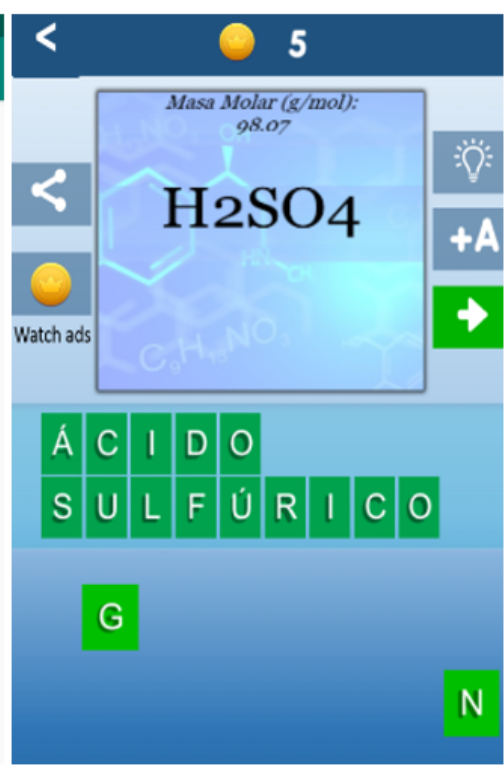

Fuente: elaboración propia

La enseñanza de los grupos funcionales en el área de química inorgánica fue desarrollada bajo la metodología de aprendizaje tradicional y la integración del Mobile learning. El propósito del empleo de ambas formas de enseñanza fue establecer si el uso de las TIC, particularmente la integración de las Apps en dispositivos móviles contribuye a mejorar el aprendizaje de los educandos. Se realizaron sesiones de trabajo, las cuales se enmarcaron en las etapas del proceso pedagógico: antes, durante y después; en esta última en cada sesión se evaluó el aprendizaje del tema explicado, los resultados se muestran en los cuadros 5 y 6 . 


\section{Cuadro 5}

Desempeño escolar área de química inorgánica estudiantes de grado décimo empleo método tradicional de aprendizaje

\begin{tabular}{ccc}
\hline Sesiones de trabajo & $\begin{array}{c}\text { Promedio desempeño académico } \\
\text { estudiantes grado décimo }\end{array}$ \\
\hline Sesión 1 & 3.3 & desempeño básico \\
\hline Sesión 2 & 2.9 & desempeño bajo \\
\hline Sesión 3 & 2.8 & desempeño bajo \\
\hline Sesión 4 & 2.5 & desempeño bajo \\
\hline Promedio sesiones & 2.87 & desempeño bajo \\
\hline
\end{tabular}

Fuente: elaboración propia

Cuadro 6

Desempeño escolar área de química inorgánica estudiantes de grado décimo empleo de Mobile Learning como estragia de aprendizaje

\begin{tabular}{ccc}
\hline Sesiones de trabajo & $\begin{array}{c}\text { Promedio desempeño académico } \\
\text { estudiantes grado décimo }\end{array}$ \\
\hline Sesión 1 & 3.9 & desempeño básico \\
\hline Sesión 2 & 4.3 & desempeño alto \\
\hline Sesión 3 & 4.0 desempeño alto \\
\hline Sesión 4 & 4.7 desempeño superior \\
\hline Sesión 5 & 4.5 desempeño alto \\
\hline Sesión 6 & 4.2 desempeño alto \\
\hline Promedio sesiones & 4.26 desempeño alto \\
\hline & Fuente: elaboración propia
\end{tabular}

Con base en el desarrollo de las sesiones de clase desde cada enfoque de enseñanza, se pudo evidenciar que cuando se desarrollaron temáticas de los grupos funcionales inorgáncos a través de la metodología tradicional, es decir, el docente en el aula de clase como transmisor de información desde la catedra magistral y el empleo de recursos didácticos como tablero y libros, el desempeño escolar de los estudiantes en las sesiones realizadas tuvo un promedio bajo, la calificación promedio alcanzada fue de 2.87 en la escala de 1.0 a 5.0. La motivación de los estudiantes no alcanzo niveles altos por la monotonia del método empleado, el desarrollo de actividades se limito a intentar dar solución a los cuestionamientos realizados por el profesor, el aprendizaje fue temporal, pues los estudiantes emplearon la memorización para tratar de retener las ideas expresadas por el docente.

En contraste con el método de aprendizaje tradicional la integración del Mobile Learning, permitió a los estudiantes desarrollar las actividades de clase de manera colaborativa. Esto propició el intercambio de información, los estudiantes se motivaron al emplear las aplicaciones Apps en el celular. El uso de estas tecnologías además les permitió recabar información de la red para fortalecer la explicación del docente, asimismo, los educandos reforzaron su conocimiento en el tema de nomenclatura de compuestos químicos inorgánicos, que es uno de los temas más complejo para ellos por la manera en que son nombrados, pues existen tres clases de nomenclatura desarrollada por la IUPAC (Unión internacional de química pura y aplicada): Tradicional, sistemática y stock. La actitud de los estudiantes con el empleo del Mobile Learning fue opuesta a la del empleo de la educación tradicional, se mostraron mas activos, participativos y dispuestos al trabajo en el aula escolar, así, por ejemplo el tiempo de clase se terminó y no se percataron de ello por estar concentrados en el desarrollo de las actividades propuestas. 
Se pudo establecer que el aprendizaje tuvo mejoras significativas como lo muestra el cuadro 6 donde se evidencia que el promedio en las calificaciones de las sesiones realizadas con el empleo del Mobile Learning alcanzó un nivel de desempeño alto, con una calificación promedio de 4.26 puntos en la escla de 1.0 a 5.0, la correlación de las variables en estudio fue positiva, a mayor empleo del Mobile Learning como estrategia de aprendizaje se evidenció mejor desempeño académico.

\subsection{Etapa de reflexión}

En esta etapa se buscó establecer si el rendimiento escolar de los estudiantes presentó mejoras significativas luego de integrar el Mobile Learning como estrategia didáctica. Se contrastaron los resultados del pre test aplicado en la etapa diagnóstica frente a la aplicación de una prueba o evaluación final o prueba post test de todos los temas desarrollados. Para realizar la comparación de datos se empleó la estadística inferencial, inicialmente se realizaron pruebas de normalidad (Shapiro-Wilk) de las calificaciones obtenidas en los temas de grupos funcionales inorgánicos por parte de los estudiantes de grado décimo, para validar supuestos de la diferencia de medias (pruebas t). Con un nivel de significancia del $5 \%(\alpha=0.05)$, los resultados del test de normalidad de datos son:

\section{Cuadro 7}

Test de normalidad

Shapiro-Wilk

\begin{tabular}{ll}
\hline Puntaje & $\begin{array}{l}\text { Estadística de Prueba: P - } \\
\text { valor }\end{array}$ \\
\hline Antes & $\mathrm{W}=0.90496, \quad \mathrm{p}$-valor $=$ \\
& 0.01113 \\
\hline Después & $\mathrm{W}=0.95027, \mathrm{p}$-valor $=0.1719$
\end{tabular}

Fuente: (elaboración propia)

Se acepta la hipótesis de normalidad en el puntaje obtenido en el post test y se rechaza en las calificaciones antes de la intervención con el Mobile Learning, es decir la fase de diagnóstico ya que $\alpha>0.01113$, por tanto, no se validan los supuestos, en tal sentido no se puede aplicar la prueba paramétrica T de Student, razón por la cual se debe aplicar la prueba no paramétrica equivalente que en este caso es el test de Wilcoxson.

\subsection{Test de prueba de los rangos con signo de Wilcoxon}

El objetivo es verificar a través de esta prueba, si hay diferencias significativas de la puntuación o calificación de los estudiantes antes y después de la intervención del Mobile Learning en la comparación del desempeño académico en el área de química inorgánica. Se considera un nivel de significancia del $5 \%(\alpha=0.05)$, es decir un nivel de confianza: 95\%. Los puntajes obtenidos del sistema de calificaciones de la institución educativa corresponden a : Diagnóstico pres test y Calificación después de la intervención con el Mobile Learning como estrategia didáctica.

\subsection{Hipótesis:}

- Ho: Los puntajes obtenidos en el diagnóstico de conocimientos inherentes a grupos funcionales inorgánicos por parte de los estudiantes de grado décimo son iguales a los obtenidos después del empleo del Mobile Learning como estrategia didáctica en el área de química. 
- Ha: Los puntajes obtenidos en el diagnóstico de conocimientos inherentes a grupos funcionales inorgánicos por parte de los estudiantes de grado décimo son diferentes a los obtenidos después del empleo del Mobile Learning como estrategia didáctica en el área de química.

Estadística de prueba: $\quad V=0, \quad p$-valor $=1.812 \mathrm{e}-06$

Decisión: Se rechaza Ho, ya que ( $p$-valor $<\alpha ; 1.812 \mathrm{e}-06<0.05)$

Conclusión: Con un nivel de significancia del 5\%, y un intervalo de confianza del 95\% hay evidencia estadística suficiente para determinar que los puntajes obtenidos en el diagnóstico son diferentes a los obtenidos después de la intervención didáctica a través del empleo del Mobile Learning, en el aprendizaje del área de química inorgánica, por parte de estudiantes de grado décimo pertenecientes al colegio Guillermo León Valencia del municipio de Duitama, Boyacá, Colombia.

\section{Gráfico 1}

Rendimiento académico pre test y post test área de química inorgánica

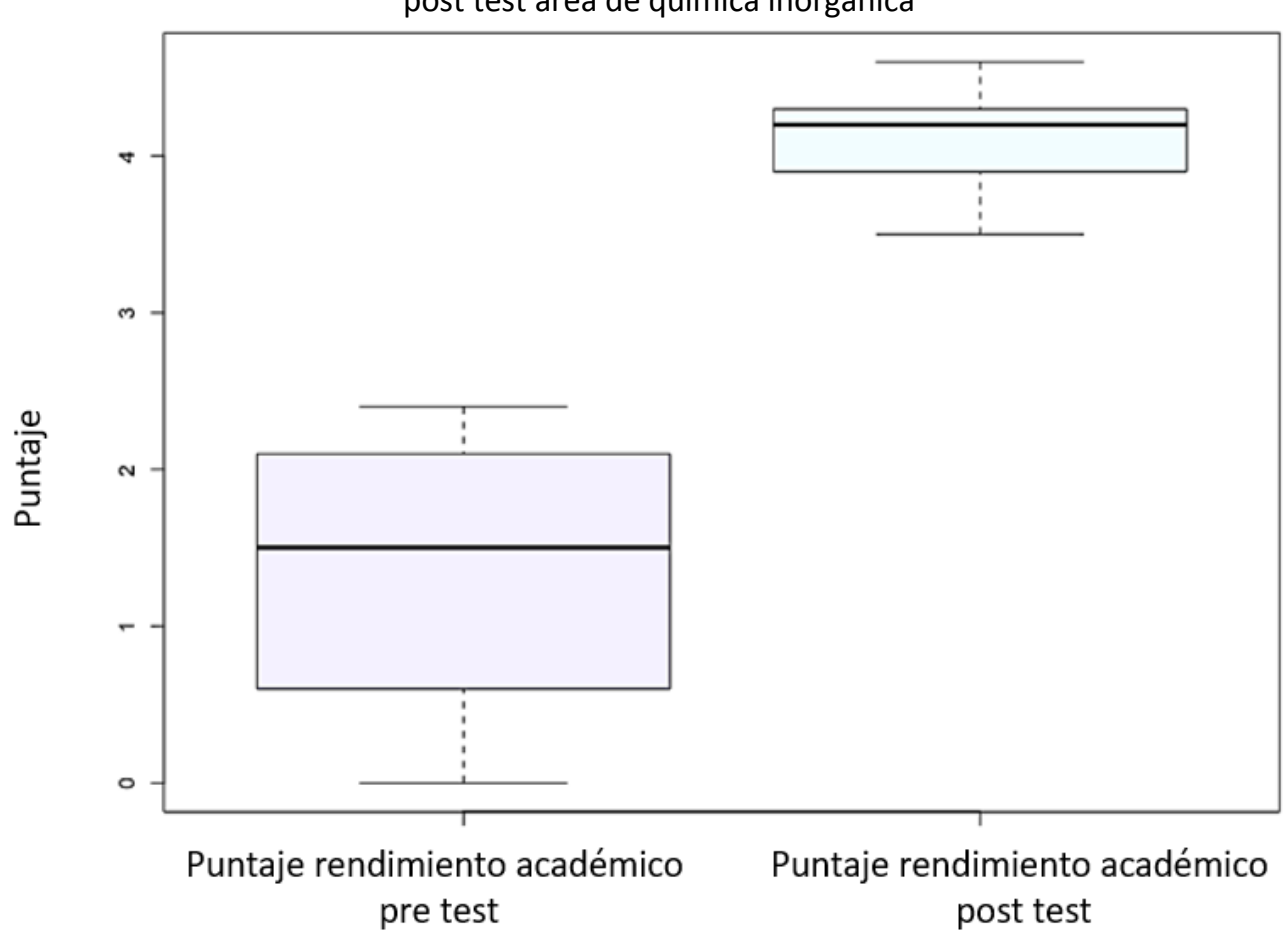

Fuente: (elaboración propia)

\section{Cuadro 8}

Resumen descriptivo puntuaciones fase post test

\begin{tabular}{cccccc}
\hline Min. & 1 cuartil. & Mediana & Media & 3 cuartil & Máximo \\
\hline 3.5 & 3.9 & 4.2 & 4.133 & 4.3 & 4.6 \\
\hline \multicolumn{5}{c}{ Fuente: elaboración propia }
\end{tabular}

Se pudo establecer que luego del proceso de intervención con integración del Mobile Learning como estrategia didáctica para la enseñanza del área de química inorgánica, los estudiantes fortalecieron el conocimiento de los grupos funcionales inorgánicos. Más del $50 \%$ de ellos obtuvieron puntajes superiores a 4.0. Con base en estos resultados se valida la hipótesis nula propuesta en la investigación: 
Hi: El uso del Mobile Learning como estrategia de enseñanza en el área de química inorgánica de grado décimo, permite que los educandos sientan agrado por los contenidos desarrollados y tengan un mejor rendimiento académico, igualmente se establece que a mayor empleo de esta metodología de enseñanza - aprendizaje, los estudiantes obtienen mejor rendimiento académico, es decir la correlación de las variables de estudio es positiva pues al aumentar una aumenta la otra.

Los estudiantes participantes en el estudio refirieron que a partir de la experiencia realizada con el empleo del Mobile learning, se sienten en capacidad de participar en las actividades desarrolladas en el área de química, que su posición no es pasiva sino que ellos aportan ideas y reflexionan frente a las situaciones planteadas, por otra parte refieren que su rendimiento académico ha mejorado significativamente, en relación al desarrollo de actividades bajo la metodología de aprendizaje tradicional, donde el docente actúa como transmisor de información y limita la participación en el desarrollo de las clases.

\section{Conclusiones}

Las conclusiones emergentes se orientan al cumplimiento de los objetivos del estudio y al desarrollo de la experiencia educativa, en este sentido se concluye que:

La integración del Mobile Learning como estrategia en el proceso pedagógico permite que el aprendizaje de los estudiantes sea interactivo, la integración de las tecnologías de la información y la comunicación en el proceso educativo, hace que se reinterpreten y resignifiquen los métodos de aprendizaje arraigados por mucho tiempo en el escenario educativo, con estas tecnologías el aprendizaje de los estudiantes no se torna monótono y sin la suficiente significación que debe tener para que verdaderamente el estudiante adquiera el conocimiento y se pueda decir que aprendió. La diversidad de aplicaciones para dispositivos móviles Apps, brindan al educando la posibilidad de aprender a su propio ritmo al tener en cuenta sus particularidades, procesos que en la educación tradicional resultan algo dispendiosos.

Cuando mayor sea el empleo del Mobile Learning como estrategia de enseñanza - aprendizaje, mejores son los resultados académicos que pueden alcanzar los estudiantes, dado que esta metodología es flexible y permite que sea el estudiante autónomo y proactivo en su proceso de formación.

El empleo de las TIC por parte del docente bajo cualquier modalidad o método, se debe orientar a mejorar sus conocimientos de estas tecnologías, el educador debe tener una formación en su empleo didáctico, pues el acceso a infraestructura no es suficiente para utilizarlas en los procesos pedagógicos y mejorar su desempeño profesional, por tal razón, los docentes deben asumir un papel protagónico a través de la exploración de diferentes programas y herramientas que les permitan dinamizar el proceso pedagógico con sus estudiantes, en este sentido, si se trata de involucrar las TIC para fortalecer el aprendizaje de los estudiantes, el docente debe ser un agente activo que permita integrar estas tecnologías para tal propósito, y no negarse a los beneficios de la tecnología, como en situaciones en las cuales se prohíbe el empleo del celular con fines educativos.

Finalmente se concluye que la experiencia realizada se cataloga de innovación, pues se demostró mejoras en el aprendizaje y rendimiento académico de los estudiantes, razón por la cual puede ser replicada a otras áreas del currículo escolar e igualmente a otros centros educativos en los cuales se presente dificultades en el aprendizaje del área de química.

\section{Referencias bibliográficas}

Arce, R. (2013). Mobile learning: aprendizaje móvil como complemento de una estrategia de trabajo colaborativo con herramientas Web 2 y entorno virtual de aprendizaje WebUNLP en modalidad de blended learning. I Jornadas Nacionales de TIC E Innovación En El Aula 
Brazuelo, F., y Gallego, D. J. (2011). Mobile learning . Los dispositivos móviles como recurso educativo. Sevilla: Mad S.L.

Cabero, J. (2007). El vídeo en la enseñanza y formación, en CABERO, J. (cood): Nuevas tecnologías aplicadas a la educación, Madrid, McGraw-Hill, 129-149. 34

Castaño, C., y Cabero, J. (2013). Enseñar y aprender en entornos M-Learning. Madrid: Editorial Síntesis.

Cantún, G. (1999). Una estrategia didáctica para el aprendizaje de la nomenclatura química inorgánica en el nivel medio superior. Universidad Autónoma de Nuevo León, San Nicolas de los Garza, Mexico

Chamocho,F(2016). Mobile Learning:nuevas posibilidades, nuevos retos. Máster en profesor de Educación Secundaria Obligatoria Y Bachillerato. Formación Profesional y Enseñanza de Idiomas. Especialidad de Tecnología e Informática. Recuperado de http://uvadoc.uva.es/handle/103

Deboer, G.E.(2000). "Scientific Literacy: Another Look at its Historical and Contemporary Meanings and its Relationship to Science Education Reform" Journal of Research in Science Teaching, 37 (6), 582-601.

Depetris, M. R., Tavela, M., \& Castro, M. F. (2012). El futuro de las tecnologías móviles y su aplicación al aprendizaje: Mobile Learning. Universidad Nacional Del Noroeste de La Provincia de Buenos Aires.

Díaz, F. (2010). Los profesores ante las innovaciones curriculares. Revista Iberoamericana de Educación Superior (RIES). Recuperado de http://ries.universia.net/index.php/ries/article/view/35/innova

Fernández, J.A., y Moreno, J.I.(2008). La química en el aula: entre la ciencia y la magia. Revista Murciencia en Researchgate. Recuperado de

https://www.researchgate.net/publication/39745810_La_Quimica_en_el_aula_entre_la_ciencia_y_la_magia

Furió, C. (2006). La motivación de los estudiantes y la enseñanza de la Química. Una cuestión controvertida.Educación química. Vol. (17), 222-227.

Hernández, G. y Montagut, P. (1991). ¿Qué sucedió con la magia de la Química?. Universidad Nacional Autonoma de México. Revista de la Educación Superior. Vol.20 N 77. Disponible en

http://www.anuies.mx/servicios/p_anuies/publicaciones/revsup/res077/txt7.htm

Izquierdo, M. (2004). Un nuevo enfoque de la enseñanza de la Química: contextualizar y modelizar. The Journal of the Argentine Chemical Society (92) 2004, pp. 115-136.

Kearney, M., Schuck, S., Burden, K., y Aubusson, P. (2012). Viewing mobile learning from a pedagogical perspective. Research in Learning Technology, 20(1), 1-17.

Nakamatsu, J. (2012). Reflexiones sobre la enseñanza de la Química. Revista de investigación. En Blanco y Negro Vol. $3 \mathrm{~N}^{\circ} 2$ - ISSN: 2221-8874. Disponible en http://revistas.pucp.edu.pe/index.php/enblancoynegro/article/download/3862/pdf/

Ng, W., y Nicholas, H. (2013). A framework for sustainable mobile learning in schools. British Journal of Educational Technology, 44(5), 695-715.

Quinn, C. (2000). mLearning: mobile, wireless, In-Your-Pocket Learning. Line Zine.

Turunen, H., Syvaenen, A., y Ahonen,M. (2003). Supporting observation tasks in a primary school with the help of mobile devices. In K Nyvri (ed) Mobile learning: essays on philosophy, psychology and education. Communications in the 21st Century. Vienna: Passage. Verlag, 209-221.

Esta obra está bajo una Licencia Creative Commons Attribución-NoCommercial 4.0 International 